\title{
Normabschätzungen für die Inversen von. Toeplitz-Matrizen
}
A. РомP

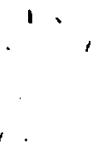

Für diskrete und paárige diskrete Wiener-Hopfsche Gleichungen vom Normaltyp, deren Symbol eine quadratisch summierbare erste Ableitung besitzt, werden die in den Fehlerabschätzungen für das Reduktionsverfahren auftretenden Konstanten abgeschätzt.

Для дисқретных и парных дискретных уравнений Винера-Хопфа әллиптического типа, символ которых имеет квадратично суммируемую первую производную, оцениваются константы, возникающие в оценках погрешности метода редукции.

For discrete and paired discrete Wiener-Hopf equations of normal type, the symbol of which possesses a quadratic summable first derivative, we estimate the constants arising in error estimates for the reduction method.

\section{Einleitung}

Für die praktische Rechnung mit Toeplitz-Matrizen

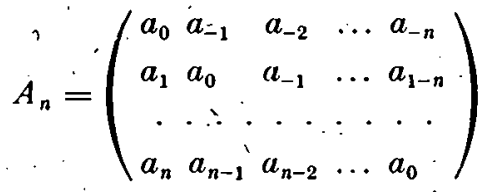

und paarigen Toeplitz-Matrizen

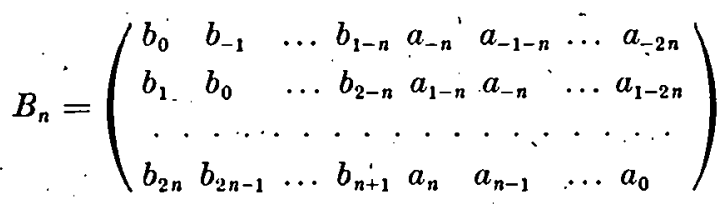

sowie

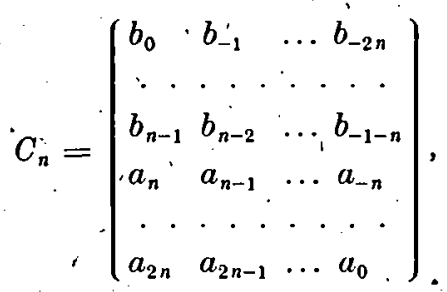

wie sie z. B. bei der Diskretisierung von Faltungsgleichungen und singulären Integralgleichungen auftreten, spiélen solche Fragen wie die der Existenz der Inversen und einer Abschätzung der Konditionszahl bzw. der Norm der Inversen eine große Rolle. 
Im Abschnitt 2 geben wir eine.Ubersicht über diejenigen Fälle, in denen Normabschätzungen für die Inversen oder wenigstens Existenzaussagen bereits bekannt sind. ${ }^{1}$ ) Nach den vórbereitenden Abschnitten 3,4,5 und 6 gelangen wir in den Abschnitten 7 und $\grave{8}$ zu Existenzaussagen ünd Normabschätzungen für. $A_{n}{ }^{-1}, B_{n}^{-1}$ und $C_{n}{ }^{-1}$ unter folgenden Vorauss̀etzungen:

1. Es existiert eine Funktion $A(z)=\sum_{-\infty}^{\infty} a_{k} z^{k}$ bzw. ein Funktionenpaar $A(z), B(z)$ $=\sum_{-\infty}^{\infty} b_{k} z^{k}$, deren Fourierkoeffizienten mit den entsprechenden Matrixelementen übereinstimmen.'

2. $A(z)$ bzw. $A(z)$ und $B(z)$ besitzen auf dem Einheitskreis $|z|=1$ eine quadratisch summierbare erste Ableitung, keine Nullstellen und den funktionentheoretischen Index Null.

Für das Reduktionsverfahren zur näherungsweisen Lösung diskreter Wiener-HopfGleichungen mit dem Sýmbol $A(z)$ und paariger diskreter Wiener-Hopf-Gleichungen mit dem Funktionenpaar $A(z), B(z)$ als Symbol werden unter obigen Voraussetzungen Fehlerabschätzungen angegeben.

Die zugrunde liegende Methode besteht im Aufstellen expliziter Formeln für. $A_{n}^{-1}, B_{n}^{-1}, C_{n}^{-1}$, wobei gewisse, durch Faktorisierungen entstandene Terme auftreten, die im allgemeinen als unbekannt angesehen werden müssen, deren Normen sich jedoch abschätzen lassen.

Die vorliegende Arbeit stellt eine Weiterentwicklung eines Teiles der'Dissertation [4] des Autors dar.

\section{Einige klassische Resultate}

Es sei $A(z)$ eine auf dem Einheitskreis $\Gamma:=\left\{e^{i \varphi} \mid 0 \leqq \varphi \leqq 2 \pi\right\}$ definierte komplexwertige, Lebesgue-integrierbare Funktion, deren Fourierkoeffizienten

$$
a_{k}=\int_{0}^{2 \pi} A\left(e^{i \varphi}\right) e^{-i k \varphi} d \varphi
$$

für $k=0, \pm 1, \ldots, \pm n$ mit den Elementen der Toeplitz-Matrix $A_{n}$ überéinstimmen. $\therefore$ Bezeichnet $u=\left\{u_{0}, u_{1}, \ldots, u_{n}\right\}^{r}$ einen beliebigen Spaltenvektor komplexer Zahlen, so gilt für die quadratische Form

$$
\left(A_{n} u, u\right):=\sum_{j=0}^{n} \sum_{k=0}^{n} a_{j-k} u_{k} \frac{1}{u_{j}}
$$

bekanntlich die Darstellung [3]:

$$
\left(A_{n} u, u\right)=\frac{1}{2 \pi} \int_{0}^{2 \pi} A\left(e^{i \varphi}\right)\left|u_{0}+u_{1} e^{i \varphi}+\cdots+u_{n} e^{i n \varphi}\right|^{2} d \varphi .
$$

Fạlls die wesentliche abgeschlossen-kovexe Hülle der Bildmenge von $A(z)$ den Null- punkt der komplexen Ebene nicht enthält oder, was dasselbe ist, wenn ein Dreh-

1) Derartige Fragestellungen werden auch in [7] behandelt, wie durch ein Referat im Zentralblatt für Math. u. ihre Grenzgebiete, Bd. 452 (1981) angekündigt wurde: Leider war uns diese Arbeit bisher nicht zugänglich. 
winkel $\psi \in[0,2 \pi]$ existiert, so daß

$$
M:=\underset{\varphi \in[0,2 \pi]}{\operatorname{ess} \inf } \operatorname{Re}\left[e^{i \varphi} A\left(e^{i \varphi}\right)\right]>0
$$

erfüllt ist, dann fọlgt aus (4):

$$
\left|\left(A_{n} u, u^{2}\right)\right| \geqq M \sum_{k=0}^{n}\left|u_{k}\right|^{2} .
$$

Folglich sind die Toeplitz-Matrizen $A_{n}$ in diesem Fall für alle $n \geqq 0$ invertierbar und für die $l^{2}$-Norm gilt die Abschätzung $\left\|A_{n}{ }^{-1}\right\| \leqq M^{-1}$. Für.reellwertige Funktionen $A(z)$ läbt sich durch Untersuchungén des asymptotischen Verhaltens der Eigenwerte von $A_{n}$ sogar $\lim _{n \rightarrow \infty}\left\|A_{n}^{-1}.\right\|=M^{-1}$ nachweisen [3].

Wenn die Bedingung (5) nicht erfüllt ist, so gilt die Aussage, da $3 A_{n}^{-1}$ für alle $n \geqq 0$ existiert, i. a. nicht mehr. Ist $A(z)$ stetig auf $\Gamma$, so sind die Bedingungen

$$
\left.\begin{array}{c}
A(z) \neq 0 \quad \forall z \in \Gamma, \\
\text { ind } A(z):=\frac{1}{2 \pi} \int_{0}^{2 \pi} d \arg A\left(e^{i \varphi}\right)=0
\end{array}\right\}
$$

hinreichend dafür, da $\beta$ eine natürliche Zahl $n_{0}$ existiert, so da $\beta$ für alle $n \geqq n_{0}$ die Matrix $A_{n}$.invertierbar und $\left\|A_{n}{ }^{-1}\right\|$ bezüglich $n$ gleichmäßig beschränkt ist [2].

Falls $A(z)$ und $B(z)$ stetige Funktionen auf $\Gamma$ sind, deren Fourierkoeffizienten mit den entsprechenden 'Koeffizienten der paarigen Toeplitz-Matrizen $B_{n}, C_{n}$ übereinstimmen, so sind die Bedingungen

$$
\left.\begin{array}{rl}
\because B(z) & \neq 0 \\
\text { ind } B(z) & =0
\end{array} \quad \forall z \in \Gamma\right\}
$$

zusammen mit den Bedingungen (6) hinreichend für die Existenz zweier natürlicher Zahlen $n_{1}, n_{2}$, so daß $B_{n}$ für alle $n \geqq n_{1}$ und $C_{n}$ für alle $n \geqq n_{2}$ invertierbar und die $l^{2}$-Normen $\left\|B_{n}{ }^{-1}\right\|,\left\|C_{n}{ }^{-1}\right\|$ bezüglich $n$ gleichmäßßig beschränkt sind [2].

Die folgenden Aussagen über die Zahlen $n_{0}, n_{1}, n_{2}$ sind seit längerem bekannt und lassen sich leicht beweisen (siehe Abschnitt 7 und 8 ):

a) Erfüllt die Funktion $A(z)$ die Bedingungen (6) und besitzt die inverse Funktion $A^{-1}(z)$ eine abbrechende Fourierreihe, also gilt

$$
A^{-1}(z)=\sum_{k=-\infty}^{m} \alpha_{k} z^{k} \quad \text { oder } A^{-1}(z)=\sum_{k=-m}^{\infty} \alpha_{k} z^{k}
$$

( $m \geqq 0$, ganz), so ist $n_{0} \leqq m-1$.

b) Erfüllen $A(z)$ und $B(z)$ die Bedingungen (6) und (7) und besitzèn die inversen Fuǹktionen abbrechende Fourierreihen,

$$
A^{-1}(z)=\sum_{k=-m}^{\infty} \alpha_{k} z^{k} \quad \text { und } \quad B^{-1}(z)=\sum_{k=-\infty}^{m_{1}} \beta_{k^{2}} z^{k}
$$

( $m, m_{1} \geqq 0$, ganz), so ist $n_{1} \leqq \max \left(m-1, m_{1}\right)$. Durch Übergang zu den konjugiert-komplexen Funktionen und den adjungierten Operatoren erhält man eine ent:sprechende Aussage für die Matrizen der Gestalt (3). 


\section{Allgemeine Invertieringsformeln}

In einem Banachraum $B$ sei ein linearer, invertierbarer Operator $W$ und ein System von Projektoren $P_{n}$ gegeben, wobei $n$ die natürlichen Zahlen oder eine Teilmenge durchläuft. $Q_{n}$ bezeichnet die érgänzenden Projektoren $Q_{n}:=I-P_{n}$.

' Für das Projektionsverfahren zur näherungsweisen Lösụng der Gleichung

$$
W x=y
$$

welches bekanntlich darin besteht, daß man zu Näherungsgleichungèn

$$
\dot{P}_{n} W P_{n} x=P_{n} y
$$

übergeht, benötigt mañ:

1. Kriterien, wañ die Gleichung (9) eine eindeutig bestimmte Lösung $\dot{x}^{(n)} \in$ im $P_{n}$ besitzt, d. h. Kriterien, wann der Operator $P_{n} W P_{n}$ im Bildraum im $P_{n}$ eine (mit $\left(P_{n} W P_{n}\right)^{(-1)}$ bezeichnete) Inverse besitzt;

2. Aussagen, unter welchen Bedingungen $x^{(n)}$ gegen, die Lösung $x$ der Ausgangsgleichung (8) konvergiert;

3. Abschätzungen für den Fehler $x-x^{(n)}$.

Zur Beantwortung dieser Fragen leistet der folgende Satz 1 oftmals nützliche Dienste. Die 'zugrunde 'liegende Idee des Ausnutzens der. Äquivalenz: $\exists\left(P_{n} W P_{n}\right)^{(-1)}$ $\Leftrightarrow \exists\left(Q_{n} W^{-1} Q_{n}\right)^{(-1)}$, geht auf A. V. Kozak zurück. (Vgl. [6].)

Es seien in $\mathfrak{B}$ zwei weitere lineare, invertierbare Operatoren $E$, $F$ gegeben, für die

$$
P_{n} E^{ \pm 1} Q_{n}=Q_{n} F^{ \pm 1} P_{n}=0
$$

gilt. Der Operator $1-F W^{-1} E$ sei als Superposition.

$$
I-F W^{-1} E=X Y
$$

darstellbar, wobè $Y$ ein linearer Òperator von $\mathfrak{B}^{\prime \prime}$ in einen Banachraum $\mathfrak{B}_{1}$ ist (der mit $\mathfrak{B}$ übereinstimmen kann) 'und $X$ ein linearer Operator von $\mathfrak{B}_{1}$ in $\mathfrak{B}$ ist. $\left(Q_{n} F W^{-1}\right.$ $\left.\times E Q_{n}\right)^{(-1)}$ bezeichnet die Inverse des Operators $Q_{n} F^{-1} E Q_{n}=Q_{n}-Q_{n} X Y Q_{n}$ im Bildraum im $Q_{n}$, falls sié existiert.

Satz 1: $\exists\left(P_{n} W P_{n}\right)^{(-1)} \Leftrightarrow \exists\left(Q_{n} F W^{-1} E Q_{n}\right)^{(-1)} \Leftrightarrow \exists\left(I-Y Q_{n} X\right)^{-1}$. Falls diese Inversen existieren, so gelten die Formeln

$$
\begin{aligned}
\left(P_{n} W P_{n}\right)^{(-1)} & =W^{-1}-W^{-1} E Q_{n}\left(Q_{n} F W^{-1} E Q_{n}\right)^{(-1)} Q_{n} F W^{-1} \\
x-x^{(n)} & =F^{-1} P_{n}\left[I-X\left(I-Y Q_{n} X\right)^{-1} Y\right] P_{n} E^{-1} \\
& =W^{-1} y-\left(P_{n} W P_{n}\right)^{(-1)} P_{n} y \\
\quad & =W^{-1} E Q_{n}\left(Q_{n} F W^{-1} E Q_{n}\right)^{(-1)} Q_{n} F Q_{n} x \\
& =W^{-1} E Q_{n}\left[I+X\left(I-Y Q_{n} X\right)^{-1} Y\right] Q_{n} F Q_{n} x
\end{aligned}
$$

Beweis: Die Formeln

$$
\begin{gathered}
\left(P_{n} W P_{n}\right)^{(-1)}=W^{-1}-W^{-1} E Q_{n}\left(Q_{n} F W^{-1} E Q_{n}\right)^{(-1)} Q_{n} F W^{-1} \\
\left(Q_{n} F W^{-1} E Q_{n}\right)^{(-1)}=E^{-1}\left[W-W P_{n}\left(P_{n} W P_{n}\right)^{(-1)} P_{n} W\right] F^{-1} \\
\left(Q_{n} F W^{-1} E Q_{n}\right)^{(-1)}=\left(Q_{n}-Q_{n} X Y Q_{n}\right)^{(-1)} \\
\quad=Q_{n}+Q_{n} X\left(I-Y Q_{n} X\right)^{-1} Y Q_{n} \\
\left(I-Y Q_{n} X\right)^{-1}=I+Y Q_{n}\left(Q_{n} F W^{-1} E Q_{n}\right)^{(-1)} Q_{n} X
\end{gathered}
$$


lassen sich auf einfache Weise direkt nachweisen,' so daß aus der Existenz der im rechtsstehenden Term auftretenden Inversen jeweils die Existenz der linksstehenden Inversen folgt. Damit ist die erste Aussage des Satzes gezeiǵt.

Aus (10), (14) und (15) ergibt.sich die Formel.(13). Es bleibt

$$
F\left(P_{n} W P_{n}\right)^{(-1)} \cdot E=P_{n}-P_{n} X\left(I-Y Q_{n} X\right)^{-1} Y P_{n}
$$

zu zeigen. Diesgeschieht, indem man den durch (15) gegebenen Ausdruck für $\left(Q_{n} F W^{-1}\right.$ $\left.\times E Q_{n}\right)^{(-1)}$ in (14) einsetzt und ausmultipliziert. Der Satz ist damit bewiesen

\section{Dárstellung der Matrizen als Operatoren}

Im Hilbertraum $l^{2}$ aller quadratisch summierbaren, beidseitigen Zahlenfolgen $\xi=\left\{\xi_{j}\right\}_{j-\infty}^{\infty}$ bezeichnen wir mit $I$ den identischen Operator, mit $P, P_{n}, Q, \dot{Q}_{n}$ die Projektoren

$$
\begin{aligned}
& P \xi:=\left\{\ldots, 0,0, \xi_{0}, \xi_{1}, \xi_{2}, \ldots\right\}, \\
& P_{n} \xi:=\left\{\ldots, 0, \xi_{-n}, \ldots, \xi_{0}, \ldots, \xi_{n}, 0, \ldots\right\},
\end{aligned}
$$

${ }^{\prime} Q:=I-P, Q_{n}:=I-P_{n}$ und mit $U^{k}(k$ ganzzahlig) die Verschiebungsoperatoren

$$
U^{k} \xi:=\left\{\xi_{j-k}\right\}_{j-\infty}^{\infty} \text {. }
$$

Offensichtlich gilt $\|P\|=\|Q\|=\left\|P_{n}\right\|=\left\|Q_{n}\right\|=\left\|U^{k}\right\|=1$. Hier wie in weiteren bedeutet die Symbolik $\|$.$\| im Falle einer Zahlenfolge oder eines Operators die Eukli-$ dische Norm, also die Norm im Raum $l^{2}$, und im Falle einer Funktion die Norm im Hilbertraum $L^{2}(\Gamma)$.

Für jede Funktion $A(z)=\sum_{-\infty}^{\infty} a_{k} z^{k} \in^{i} L^{\infty}(\Gamma)$ stellt die unendliche Matrix $\left(a_{j-k}\right)_{j, k-\infty}^{\infty}$ einen in $l^{2}$ stetigen Opertor $A$ dar, den man als Laurent-Operator bezeichnet [1]. Gehört die Funktion $A(z)$ der Wienerschen Algebra $\mathfrak{W}$ an,

$$
A(z) \in \mathfrak{W} \stackrel{\text { der }}{\Leftrightarrow} \sum_{-\infty}^{\infty}\left|a_{k}\right|<\infty
$$

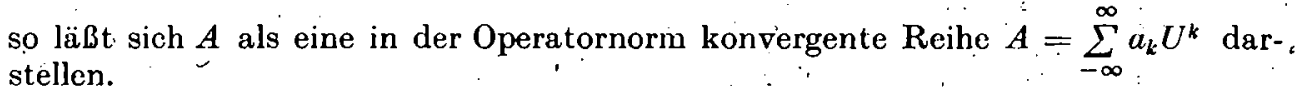
Die Toeplitz-Matrix (1) läßt sich mit dem Operator $P_{n} P A P P_{n}$ identifizieren, wobei $A(z)$ eine beliebige, absolut konvergente Fortsetzung der Reihe $\sum_{k=-n}^{\infty} a_{k} z^{k}$ ist. Die paarigen,Toeplitz-Maitrizen (2) und (3) lassen sich mit den Operatoren $P_{n}(A P+B Q) P_{n}$ bzw. $P_{n}(P A+Q B) \cdot P_{n}$ identifizieren, wobei $A(z)$ und $B(z)$ beliebige, absolut konvergente Fortsetzungen der Reihen $\sum a_{k} z^{k}$ und $\sum \cdot b_{k} z^{k}$. (mit den entsprechenden Summationsgrenzen) sind.

\section{Die Faktorisierung} Mit $R_{+}$und $R_{-}$bèzeichnen wir die für alle Funktionen $f(z)=\sum_{-\infty}^{\infty} f_{k} z^{k} \in L^{2}(\Gamma)$ définier-
ten Operatoren:

$$
R_{+} f(z):=\frac{1}{2} f_{0}+\sum_{k=1}^{\infty} f_{k} z^{k}, \quad R_{-} f(z):=\frac{1}{2} f_{0}+\sum_{k=1}^{\infty} f_{-k^{2}} z^{-k}
$$


$R_{+}$und $R_{-}$sind stetige Operatoren in $L^{2}(\Gamma)$. Offensichtlich ist $R_{+}+R_{-}=I$. Bezeichnet $H$ den Hilbertschen singulären Integraloperator

$$
\text { (Hf) }\left(e^{i \varphi}\right):=\frac{1}{2 \pi} \int_{0}^{2 \pi} \cot \frac{\sigma-\varphi}{2} f\left(e^{i \sigma}\right) d \sigma,
$$

so gilt weiterhin $H=i\left(R_{+}-R_{-}\right)$(siehe z. B. [5]). Für alle reellwertigen Funktionen $f(z) \in L^{2}(\Gamma)$ ist $R_{-} f(z)=\overline{R_{+} f(z)}$ (konjugiert-komplexer Wert). Daraus folgt für die Real- und Imaǵginärteile bei einer reellwertigen Funktion $f(z)$ :

$$
\operatorname{Re} R_{+} f(z)=\operatorname{Re} R_{-} f(z)=\frac{1}{2} f(z), \quad-\operatorname{Im} R_{+} f(z)=\operatorname{Im} R_{-} f(z)=\frac{1}{2} H f(z) .
$$

Setzen wir für eine Funktion $A(z) \in \mathfrak{W}$ das Erfülltsein der Bedingungen (6) voraus, so liegt das Bild dieser Funktion im Holomorphiegebiet der komplexen LogarithmusFunktion und folglich gilt:

$$
\ln A(z)=\ln |A(z)|+i \arg A(z) \in \mathfrak{W} \text {. }
$$

Auch die Funktionen

$$
R_{ \pm} \ln A(z)=\frac{1}{2}[\ln |A(z)| \mp i H \ln |A(z)|+i \arg A(z) \pm H \arg A(z)]
$$

gehören der Wienerschen Algebra $\mathfrak{W}$ an. Die Darstellung $A(z)=A_{-}(z) A_{+}(z)$ mit $A_{ \pm}(z)=\exp \left[R_{ \pm} \ln A(z)\right]$ bezeichnet man als Faktorisierung der Funktion $A(z)$. Es gilt $A_{ \pm}^{ \pm 1}(z) \in \mathfrak{W}$ sowie

$$
\begin{gathered}
\left|A_{ \pm}(z)\right|=\sqrt{|A(z)|} \exp \left[ \pm \frac{1}{2} H \arg A(z)\right] \\
\arg A_{ \pm}(z)=\frac{1}{2} \arg A(z) \mp \frac{1}{2} H \ln |A(z)|
\end{gathered}
$$

Es̀ läßt sich zeigen, daß alle Fourierkoeffizienten vón $A_{+}^{ \pm 1}(z)$ mit negátivem und alle Koeffizienten von $A_{-}^{ \pm 1}(z)$ mit positivem Index verschwinden. Für die zugeordneten Laurent-Operatoren $A_{+}, A_{+}^{-1}, A_{-}, A_{-}^{-1}$ gelten folglich die Beziehungen $Q A_{+}^{ \pm 1} P$ $=P A_{-} \pm 1=0$. Die Normen dieser Operatoren sind gleich dem Maximum der erzeugenden Funktionen (vgl. $[1,2])$ :

$$
\left\|A_{ \pm}\right\|=\max _{z \in \Gamma}\left|A_{ \pm}(z)\right|, \quad\left\|A_{ \pm}{ }^{-1}\right\|=\max _{z \in \Gamma}\left|A_{ \pm}{ }^{-1}(z)\right|
$$

\section{Einige Bezeichnungen, und Abschätzungèn}

Fis sei $A(z) \in \mathfrak{W}$ eine Funktion, die den Bedingungen (6) genügt. Wir führen folgende Bezeichnungen eị:

$$
\begin{aligned}
& \min |A(z)|:=\min _{z \in \Gamma}|A(z)|, \quad \max |A(z)|:=\max _{z \in \Gamma}|A(z)|, \\
& h(A):=\exp \left(\max _{z \in \Gamma} \mid H \arg A(z) \|\right), \\
& \gamma_{0}(A):=\frac{1}{2 \pi} \int_{0}^{2 \pi} \arg A\left(e^{i_{\varphi}}\right) d \varphi, \\
& g(A):=\max \left(\left\|A_{+}\right\|,\left\|A_{-}\right\|\right)
\end{aligned}
$$


Aus (16) und (17) folgt

$$
\begin{aligned}
& g(A) \leqq \sqrt{h(A) \max |A(z)|}, \\
& g\left(A^{-1}\right)=\max \left(\left\|A_{+}{ }^{-1}\right\|,\left\|A_{-}^{-1}\right\|\right) \leqq \sqrt{\frac{h(A)}{\min |A(z)|}}
\end{aligned}
$$

Falls die Bedingung

$$
\frac{d}{d \varphi} A\left(e^{i \varphi}\right) \in L^{2}(\Gamma)
$$

erfüllt ist, so setzen wir

$$
\mu(A):=\left\|\frac{A^{\prime}(z)}{A(z)}\right\| \exp \left(\left\|\arg A(z)-\gamma_{0}(A)\right\|\right)
$$

Wir erinnern daran, daß $\|\cdot\|$ im Falle einer Funktion die Norm in $L^{2}(\Gamma)$ und im Falle eines Operators die Norm in $l^{2}$ bedeutet.

Für eine Abschätzung der Zahl $h(A)$ gibt es mehrere Möglichkeiten. Genügt arg $(A z)$ einer Lipschitz-Bedingung.

$$
\left|\arg A(z)-\arg A\left(z^{\prime}\right)\right| \leqq K\left|z-z^{\prime}\right| . \forall z, z^{\prime} \in \Gamma
$$

so gilt [5]:

$$
|H \arg A(z)| \leqq \frac{4 K}{\pi} \Rightarrow h(A) \leqq \exp \left(\frac{4 K}{\pi}\right) .
$$

Genügt $A(z)$ den Bedingungen (6) und (18), so besitzt auch die Funktion arg $A(z)$ eine quadratisch summierbare erste Ableitung. Bezeichnet $\arg A(z)=\sum_{-\infty}^{\infty} \gamma_{k} z^{k}$ (mit $\left.\gamma_{-k}=\overline{\gamma_{k}}, \gamma_{0}=\gamma_{0}(A)\right)$ die Fourierreihe dieser. Funktion, so folgt für $h(A)$ die Abschätzung:

$$
\ln h(A) \leqq 2 \sum_{1}^{\infty}\left|\gamma_{k}\right| \leqq 2 \sqrt{\sum_{k=1}^{\infty} k^{-2}} \sqrt{\sum_{k=1}^{\infty} k^{2}\left|\gamma_{k}\right|^{2}}=\frac{\pi}{\sqrt{3}}\left\|\frac{d}{d \varphi} \arg A_{k}\left(e^{i \varphi}\right)\right\| .
$$

Hilfssatz 1: Unter den Voraussetzungen (6) und (18) gelten für $n=0,1,2, \ldots$ die Abschätzungèn :

$$
\begin{aligned}
& \left\|P Q_{n} A_{+}^{-1} A_{-} Q\right\| \leqq(n+3 / 2)^{-1 / 2} \mu(A), \\
& \left\|Q A_{+} A^{-1} P Q_{n}\right\| \leqq(n+3 / 2)^{-1 / 2} \mu(A) \\
& \| P A_{+}^{-1} A_{-} Q Q_{n} \leqq(n+1 / 2)^{-1 / 2} \mu(A) \\
& \left\|Q Q_{n} A_{+} A_{-}^{-1} P\right\| \leqq(n+\cdot-1 / 2)^{-1 / 2} \mu(A)
\end{aligned}
$$

Beweis: Zunächst sei bemnerkt, daß aus (18) $A(z) \in \mathfrak{B}$ folgt. Setzen wir

$$
e^{i H \ln A(z)}=A_{+}^{-1}(z) A_{-}(z)=: D(z)=\sum_{k=-\infty}^{\infty} d_{k} z^{k} \in \mathfrak{W},
$$


so folgt aus

$$
\begin{aligned}
\left\|D^{\prime}(z)\right\| & =\left\|i D(z) \frac{d}{d \varphi} H \ln A\left(e^{i \varphi}\right)\right\| \leqq\|D(z)\|\left\|H \frac{d}{d \varphi} \ln A\left(e^{i \varphi}\right)\right\| \\
& =\left\|e^{-\operatorname{Im} H \ln A(z)}\right\|\left\|\frac{A^{\prime}(z)}{A(z)}\right\|=\left\|e^{-H \arg \lambda_{(z)}}\right\|\left\|\frac{A^{\prime}(z)}{A(z)}\right\| \leqq \mu(A),
\end{aligned}
$$

und aus $P U^{k} Q \dot{Q}_{n} \doteq 0$ (für $k \leqq n$ ) für die-linke Seite der-Ungleichung (21) die Abschätzung:

$$
\begin{aligned}
\left\|P A_{+}^{-1} A_{-} Q Q_{n}\right\| & =\left\|P \sum_{k=n+1}^{\infty} d_{k} U^{k} Q Q_{n}\right\| \leqq \sum_{k=n+1}^{\infty}\left|d_{k}\right| \\
& \leqq \sqrt{\sum_{k=n+1}^{\infty} k^{-2}} \sqrt{\sum_{k=n+1}^{\infty} k^{2}\left|d_{k}\right|^{2}} \leqq \sqrt{\int_{n+1 / 2}^{\infty} t^{-2} d t}\left\|D^{\prime}(z)\right\| \\
& \leqq(n+1 / 2)^{-1 / 2} \mu(A) .
\end{aligned}
$$

Analog zeigt man (19), (20) und (22). Hilfssatz 1 ist damit bewiesen

\section{Aussagen für die Matrizen $\boldsymbol{A}_{\boldsymbol{n}}$ und die Operatoren $\boldsymbol{P A P}$}

Es sei $A(z)=\sum_{-\infty}^{\infty} a_{k} z^{k}$ eine Funktion, die den Bedingungen (6) und (18) genügt, und $A$ der zugeordnete Laurent-Operator. Für die Schar der durch $A(z)$ erzeugten Toeplitz-, Matrizen $A_{n}{ }^{\prime}(n=0,1,2, \ldots)$ und für das Reduktionsverfahren zur näherungsweisen Lösung der diskreten Wiener-Hopf-Gleichung

$$
P A P x=y^{\prime} \quad(y \in \operatorname{im} P)
$$

im Raum ${l_{+}}^{2}=$ im $\boldsymbol{P}$ lássen sich die im nachstehenden Satz 2 genannten Aussagen treffen. Interessiert man sich lediglich für ein Invertierbarkeitskriterium und die Inversen-Abschätzung einer einzelnen Matrix der Gestalt (1), so kann man unter allen, die Bedingungen (6) und (18) erfüllenden Fortsetzungen der Reihe $\sum^{n} a_{k} z^{k}$ eine solche auswählen, mit der man möglichst günstige Aussagen erhält:

Satz 2: Aus

$$
\vartheta_{n}:=\frac{\mu(A)}{\sqrt{n+3 / 2}}<1
$$

folgt die Existenz der Inversen $A_{n}{ }^{-1}$ sowie die Abschätzung

$$
\left\|A_{n}{ }^{-1}\right\| \leqq \frac{2 h(A)}{\left(1-\vartheta_{n}\right) \min |A(z)|}
$$

Die Näherungsgleichung $P P_{n} A P P_{n}=P_{n} y$ besitzt also in diesem Fall eine eindeutig bestimmte Lösung $x^{(n)} \in$ im $P P_{n}$ und für den Abstand zur exakten Lösung der Gleichung. (23) gilt:

$$
\left\|x-x^{(n)}\right\| \leqq \sqrt{\frac{\max |A(z)|}{\min |(A z)|}} \frac{h(A)}{1-\vartheta_{n}}\left\|Q_{n} x\right\| .
$$

Beweis: Wir werden den Satz 1 anwenden. $Z$ u diesem Zweck setzen wir $\mathfrak{B}=l_{+}{ }^{2}$, $\mathfrak{B}_{1}=l^{2}, \quad W=P A P, \quad E=P A_{+} P, \quad F=P A_{-} P . \quad$ Dann $\quad$ ist $\quad X=P A_{+}{ }^{-1} A_{-} P$, 
$Y=Q A_{+} A_{-}^{-1} P, W^{-1}=P A_{+}{ }^{-1} P A_{-}^{-1} P$ und die Gleichungen (10) und (11) sind, wie man leicht nachprüfen kann, erfüllt.

Aus der Abschätzung

$$
\left\|\dot{Y} Q_{n} X\right\| \leqq\left\|Y Q_{n}\right\|\left\|Q_{n} X\right\| \leqq(n+3 / 2)^{-1} \mu^{2}(A)=\grave{\vartheta}_{n}^{2}
$$

folgt; daß für $\vartheta_{n}<1$ der Operator $I-Y Q_{n} X$ invertierbar ist, und damit ist auch der Operator $P P_{n} A P P_{n}$ im Bildraum im $P P_{n}$ invertierbar, d. h., es existiert $A_{n}{ }^{-1}$.

Die Formeln (15) und (24) liefern $\left\|\left(Q_{n} F W^{-1} b Q_{n}\right)^{(-1)}\right\| \leqq\left(1-\vartheta_{n}^{2}\right)^{-1}$. Aus (12) und den Ạbschätzungen

$$
\begin{aligned}
\left\|W^{-1} E Q_{n}\right\| & =\left\|A_{+}{ }^{-1} P A_{-}{ }^{-1} A_{+} P Q_{n}\right\|=\left\|A_{-}^{-1} P Q_{n}-A_{+}{ }^{-1} Y Q_{n}\right\| \\
& \leqq\left(1+\vartheta_{n}\right) g\left(A^{-1}\right) \\
\left\|Q_{n} F W^{-1}\right\| & =\left\|Q_{n} P A_{-} A_{+}{ }^{-1} P A_{-}^{-1}\right\| \leqq\left\|Q_{n} P A_{+}^{-1}-Q_{n} X A_{-}^{-1}\right\| \\
& \leqq\left(1+\vartheta_{n}\right) g\left(A^{-1}\right)
\end{aligned}
$$

folgt nun

$$
\left\|\left(\dot{P P_{n}} A \dot{P P} P_{n}\right)^{(-1)}\right\| \leqq g^{2}\left(A^{-1}\right)+\frac{\left(1+\vartheta_{n}\right)^{2} g^{2}\left(A^{-1}\right)}{1-\vartheta_{n}^{2}}=\frac{2 g^{2}(A-1)}{1-\vartheta_{n}}
$$

Aus (13) und obigen Abschätzungen ergibt sich schließlich

$$
\begin{aligned}
\left\|x-x^{(n)}\right\| & \leqq\left(1-\vartheta_{n}^{2}\right)^{-1}\left\|W^{-1} E Q_{n}\right\|\left\|Q_{n} F\right\| \cdot\left\|Q_{n} x\right\| \\
& \leqq \frac{g(A) g\left(A^{-1}\right)}{1-\vartheta_{n}}\left\|Q_{n} x\right\| \square \quad \therefore \quad \therefore
\end{aligned}
$$

Andere Invertierbarkeitskriterien und Abschätzungen erhält man durch eine andere Wahlder Operatoren $E$ und $F$. Nehmen wir z. B. $E=P A_{+}^{-1} P$ und $F=P A_{-}^{-1} P$, so ist

$$
Q_{n} F(P A P)^{-1} E Q_{n}=Q_{n} P A^{-2} \dot{P} Q_{n}-Q_{n} P A^{-1} Q A^{-1} P Q_{n}
$$

Dieser Operator ist invertierbar, falls

$$
\left\|Q_{n} P A^{-1} Q A^{-1} P Q_{n}\right\|\left\|\left(P A^{-2} P\right)^{-1}\right\|<1 .
$$

Bezeichnet $\sum_{-\infty}^{\infty} \alpha_{k} z^{k}$ die Fourierentwicklung der Funktion $A^{-1}(z)$, so ist die Bedingung

$$
\left|\sum_{k=n+2}^{\infty} \alpha_{-k} z^{-k}\right|\left|\sum_{k=n+2}^{\infty} \alpha_{k} z^{k}\right|\left\|A_{+}{ }^{2} P A_{-}{ }^{2}\right\|<1 .
$$

hinreichend für die Existenz von $A_{n}^{-1}$. Daraus ergibtsich unmittelbar die Aussage a aus Abschnitt 2.

Bemerkung: Durch Abschätzung der Norinen von $A_{ \pm}^{-1}(z)$ in $\mathfrak{W}$ mittels dèr Hölderschén Ungleichung lassen sich auch die $l^{p}$-Normen $(1 \leqq p \leqq \infty)$ der Matrizen $A_{n}^{-1}$ nach oben abschätzen. 


\section{Aussagen für paarige Toeplitz-Matrizen und parige diskrete Wiener-Hopf-Gleichungen}

Es séen $A(z)=\sum_{-\infty}^{\infty} a_{k} z^{k}, B(z)=\sum_{-\infty}^{\infty} b_{k} z^{k}$ zwei Funktionen, die den Bedingungen (6) und (18) genügen, $A$ und $B$ die zugeordneten Laurent-Operatoren und $A=A_{-} A_{+}$, $B=B_{-} B_{+}$ihre Faktorisierungen. Die dem Satz 2 vorangestellte Bemerkung ist sinngemäß auch hier anzuwenden, wenn man sich nur für eine spezielle Matrix der Gestalt (2)'interessiert.

Wir führen noch folgende Bezeichnungen ein:

$$
\begin{aligned}
\left.h\left(A B^{-1}\right)\right) & =\exp \{\max |H[\arg A(z)-\arg B(z)]|\} \\
g & :=h\left(A B^{-1}\right) \max \left[g\left(A^{-1}\right) g(B), g(A) g\left(B^{-1}\right)\right], \\
\delta_{n} & :=\dot{g}\left(A B^{-1}\right) \max \left[\frac{g(A) g\left(A^{-1}\right) \mu(\dot{B})}{\sqrt{n+1 / 2}}, \frac{g(B) g\left(B^{-1}\right) \mu(A)}{\sqrt{n+3 / 2}}\right] .
\end{aligned}
$$

Sa tz 3: Für $\delta_{n}<1$ existiert $B_{n}^{-1}$ und es gilt die Abschätzung

$$
\begin{aligned}
\left\|B_{n}^{-1}\right\| \leqq & \sqrt{\frac{h\left(A B^{-1}\right)}{\min \lfloor A(z) B(z) \mid}}\left\{\max \left[g(A) g\left(B^{-1}\right) h(\dot{B}), g\left(A^{-1}\right) g(B) h(A)\right]+\frac{\tilde{g} \delta_{n}}{1-\delta_{n}}\right\} \\
& +\frac{\bar{g} g\left(A^{-1}\right) g\left(B^{-1}\right)}{1-\delta_{n}} \max \left[\frac{\mu(A)}{\sqrt{n+3 / 2}}, \frac{\mu(B)}{\sqrt{n+1 / 2}}\right] .
\end{aligned}
$$

Die somit eindeutig bestimmte Lösung $x^{(n)} \in$ im $P_{n}$,der Näherungsqleichung $P_{n}(A P$ $+B Q) P_{n} x=\dot{P}_{n} y$ konvergiert für alle $y \in l^{2}$ gegen die Lösung $x$ der Gleichung $(A P$ $+B Q) x=y$ :

$$
\begin{aligned}
\left\|x-x^{(n)}\right\| \leqq & \left\{\max g\left[(A) g\left(A^{-1}\right), g(B) g\left(B^{-1}\right)\right]\right. \\
\therefore & \left.+\frac{\cdot g}{1 \div \delta_{n}} \max \left[\frac{g(A) g\left(B^{-1}\right) \mu(A)}{\sqrt{n+3 / 2}}, \frac{g\left(A^{-1}\right) g(B) \mu(B)}{\sqrt{n+1 / 2}}\right]\right\}\left\|Q_{n} x\right\| .
\end{aligned}
$$

-Beweis: Wir setzen $\mathfrak{B}=\mathfrak{B}_{1}=l^{2} ; W=A P+B Q, E=A_{+} P-B_{-} Q, F=P A_{-}$ $-Q B_{+}$. Dann gilt, wie man leicht nachprüfen kann:

$\dot{W}^{-1}=\left(A_{+}^{-1} B_{+} P+A_{-} B_{-}^{-1} Q\right) A_{-}^{-1} B_{+}^{-1}$,

$F W^{-1} E=I-\left(P A_{+}^{-1} A_{-} B_{+}+Q \dot{A}_{-} B_{+} B_{-}^{-1}\right)\left(Q A_{+} A_{-}^{-1} B_{+}{ }^{-1} P+P A_{-}^{-1} B_{+}^{-1} B_{-} Q\right)$;

$X=\left(P B_{-}+Q A_{+}\right) A_{+}^{-1} A_{-} B_{+} B_{--}^{-1} ; \quad Y=P A_{-}^{-1} P B_{+}^{-1} B_{-} Q+Q B_{+}^{-1} Q A_{+} A_{-}^{-1} P$.

Aus (16) folgt:

$$
\left\|A_{+}^{-1} A_{-} B_{+} B_{-}{ }^{-1}\right\|=\exp \left\{\max _{z \in r^{\prime}}[H \arg B(z)-H \arg A(z)]\right\} \leqq \dot{h\left(A B^{-1}\right)^{\prime} \cdot .} .
$$

Benutzen wir die Ungleichungen (20), (21) und die Tatsache, daß für zwei beliebige lineare, stetige Operatoren $\mathcal{A}, \mathscr{B}$ im Hilbertraum $l^{2}$

$$
\begin{aligned}
& \|P \mathscr{A} P+Q \mathscr{B} Q\|=\max (\|P \mathcal{A} P\|,\|Q \mathscr{B} Q\|), \\
& \|P \mathcal{A} Q+Q \mathscr{B} P\|=\max (\|P \mathscr{A} Q\|,\|Q \mathscr{B} P\|)
\end{aligned}
$$


gilt, so gelangen wir żu der Abschätzung $\left\|Y Q_{n} X\right\| \leqq \delta_{n}$. Mittels Satz 1 folgt daraus die Hinlänglichkeit der Bedingung $\delta_{n}<1$ für die Existenz von $B_{n}{ }^{-1}$. Die Fehlerabschätzung (26) erhält man mit Hilfe der aus (13) abgeleiteten Formel

$$
x-x^{(n)}=\left[F^{-1} Q_{n} F-F^{-1} P_{n} X\left(I-Y Q_{n} X\right)^{-1} Y Q_{n} F\right] Q_{n} x,
$$

aus $\left\|F^{-1} P_{n} X\right\| \leqq \tilde{g}$ und entsprechenden Abschätzungen für $\left\|F^{-1} Q_{n} F\right\|$ und $\left\|Y Q_{n} F\right\|$.

Analog ergibt sich mit.Hilfe der aus (12) abgeleiteten Formel

$$
\begin{gathered}
\left(P_{n} W P_{n}\right)^{(-1)}=F^{-1} P_{n} F W^{-1}+F^{-1} P_{n} X\left\{\left[I-\left(I \div Y \dot{Q}_{n} X\right)^{-1}\right] Y E^{-1}\right. \\
\left.\quad+\left(I-Y Q_{n} X\right)^{-1} Y Q_{n} E^{-1}\right\}
\end{gathered}
$$

und entsprechenden Abschätzungen für $\left\|F^{-1} P_{n} F W^{-1}\right\|,\left\|Y E^{-1}\right\|$ und $\left\|Y Q_{n} E^{-1}\right\|$ die Ungleichung (25). Da $Y Q_{n}$ und mithin auch der ganze zweite Summand in der Operatornorm gegen . Null konvergiert, ergeben sich auf diese Weise für große Zahlen $n$. bessere Abschätzungen, als durch direktes Abschätzen der in Formel (12) auftretenden Terme. Satz 3 ist damit bewiesen

Dúrch Umstellungen der verwendeten Formeln ergeben sich andere Möglichkeiten der Abschätzung. Wir haben' uns hier um eine Variante bemüht, in der nur solche Größen auftreten, die durch als bekannt anzusehende Zahlen abgeschätzt werden' können, und bei der die angegebenen Schranken für großes $n$ möglichst klein werden. Die Einführing der Operatoren $X$ und $Y$ diente dem Zweck, in dem Ausdruck $Y Q_{n} X$ die Norm der Summe zweier Operatoren durch das Maximum der Normen abschätzen zu können.

Weitere Möglichkeiten erhält man durch eine andere Wahl der Operatoren $E$ und $F$. Setzt man z. B: $F=P A_{-}^{-1} B_{-}+Q A_{+} B_{+}^{-1}$ und $E=A_{+}^{-1} B_{+} P+A_{-} B_{-}^{-1} Q$, so gilt für $W=A P+B Q$ :

$$
\begin{aligned}
Q_{n} F W^{-1} E Q_{n}= & Q_{n}\left(P A^{-2} B P+Q A B^{-2} Q\right) Q_{n} \\
& +Q_{n}\left(P A^{-1} B-Q A B^{-1}\right)\left(P B^{-1} Q-Q A^{-1} P\right) Q_{n}
\end{aligned}
$$

Der erste Summand auf der rechten Seite ist ein invertierbarer Operator in im $Q_{n}$ für alle $n=0,1,2, \ldots$ und die Operatoren $P B^{-1} Q Q_{n}, Q A^{-1} P Q_{n}$ konvergieren in der. Operatornorm gegén Null. Aus (27) ergibt sich auch unmittelbar die Aussage b aus Abschnitt 2.

Der Fäll $\dot{W}=P A+Q B$ läßt sich in analoger Weise behandeln. Wählt man auch hier $E=A_{+} P-B_{-} Q, F=\cdot P A_{-}-Q B_{+}$, so erhält man für $X$ und $Y$ die Ausd'rücke

$$
\begin{aligned}
& X=P A_{+}{ }^{-1} A_{-} Q B_{-}^{-1} Q+Q B_{+} B_{-}^{-1} P A_{+}{ }^{-1} P, \\
& Y \doteq A_{+} A_{-}^{-1} B_{+}{ }^{-1} B_{-}\left(B_{+} P+A_{-} Q\right)
\end{aligned}
$$

$\left\|Y Q_{n} X\right\|$ läßt sich ebenfalls durch $\delta_{n}$ abschätzen, so dá́ die Bedingung $\delta_{n}<1$ auch hinreichend für die Existenz von $C_{n}^{-1}$ ist. Abschätzungen für $C_{n}^{-1}$ und des beim Reduktionsverfahren auftretenden Fehlers kann man in ähnlicher Weise erhalten wie open. 


\section{Weitere Anwendungen}

Das Reduktionsverfahren zur näherungsweisen l. Iösung der singulärèn Integralgleichung

$$
\tilde{A}(z) x(z)+\frac{\tilde{B}(z)}{\pi i} \int_{\Gamma} \frac{x(\zeta)}{\zeta-z} d \zeta=y(z) \quad(z \in I)
$$

besteht bekanntlich darin, daß man zu Näherungsgleichungen

$$
B_{n} x^{(n)}=\left\{y_{-n}, \ldots, y_{0}, \ldots, y_{n}\right\}^{r}
$$

übergeht. Dabei ist $\left\{y_{-n}, \ldots, y_{n}\right\}^{\text {r }}$ ein Spaltenvektor mit den Fourierkoeffizienten von $y(z)$ und $B_{n}$ eine Matrix der Gestalt (2), wobei $a_{k}$ und $b_{k}$ entsprechend die Fourierkoeffizienten der Funktionen $A(z)=\tilde{A}(z)+\tilde{B}(z)$ und $B(z)=\tilde{A}(z)-\tilde{B}(z)$ sind $[(2$, 5]). Erfüllen $A(z)$ und $B(z)$ die Bedingungen (6) und (18), so läBt sich der Satz 3 anwenden. Für $\delta_{n}<1$ besitzt die Gleichung (29) eine eindeutig bestimmte Lösung $x^{(n)}=\left\{x_{-n}^{(n)}, \ldots ; x_{n}^{(n)}\right\}^{\mathbf{x}}$ und die daraus gebildete Funktionenfolge $x^{(n)}(z)=\sum_{k=-n}^{n} x_{k}^{(n)} z^{k}$ konvergiert für alle $y(z) \in L^{2}(F)$ in der Norm von $L^{2}(\Gamma)$ gegen die (eindeutig bestimute). Lösung $x(z)$ der Gleichung (28). Der Fehlèr $\left\|x(z)-x^{(n)}(z)\right\|$.läßt sich unter Ausnutzung der Parsevalschen Gleichung mittels (26) abschätzen.

Für singuläre Integralgleichungen, bei denen die Funktion $\tilde{B}(z)$ mit unter dem Integral steht, gelten, bis auf eine andere Fehlerabschätzung, die gleichen Aussagen, wenn man in der Näherungsgleichung (29) $B_{n}$ durch $C_{n}$ ersetzt.

Eine Möglichkeit zur näherungsweisen Läsung der Wiener-Hopfschen Integralgleichung

$$
(A x)(t):=x(t)-\int_{0}^{\infty} k(t-s) x(s) d s \stackrel{=}{=} y(t) \quad(0<t<\infty)
$$

stellt das Galerkinsche Verfahren "nach. dem in $L^{2}(0, \infty)$ orthonormierten System $\left\{\psi_{j}(t)\right\}_{j=0}^{\infty}$ dèr Laguerreschen Funktionen dar [2]. Die Näherungsgleichung hat hier die Gestalt

$$
\sum_{k=0}^{n} \xi_{k}\left\langle A \psi_{k}, \psi_{j}\right\rangle=\left\langle y, \psi_{j}\right\rangle \quad(j=0,1, \ldots, n)
$$

wobei $\langle\cdot,:\rangle$ das.Skalarprodukt in $L^{2}(0, \infty)$ ist. Die Koeffizientenmatrix der Gleichung (31). ist eine Toeplitz-Matrix mit der erzeugenden Funktion [2]

$$
A(z)=1-\int_{-\infty}^{\infty} k(t) \exp \left(\frac{z+1}{z-1} t\right) d t . \quad(z \in F)
$$

Genügt $A(z)$ den Bedingungen (6) und (18), so ist der Satz 2 anwendbar. Für $\vartheta_{n}<1$ besitzt (31) eine eindeutig bestimmte Lösung $\xi^{(n)}=\left\{\xi_{0}{ }^{(n)}, \ldots, \xi_{n}{ }^{(n)}\right\}$ und die Funktionen $x^{(n)}(t)=\sum_{k=0}^{n} \xi_{k}^{(n)} \psi_{k}(t)$ konvergieren für alle $y \in L^{2}(0, \infty)$ in derr Norm $\nabla p n$ $L^{2}(0, \infty)$ gegen die (eindeutig bestimmte) Lösung $x(t)$ der Gleichung (30). Unter Auśnutzung der Parsevalschen Gleichung läßt sich der Fehler $\left\|x(t)-x^{(n)}(t)\right\|_{L^{2}(0, \infty)}$ wie im Satz 2 abschätzen. 


\section{.LITER.ATUR}

[1] Brown, A., and P. R. Halsos: Algebraic Properties of Toeplitz Operators. Journ. f. reine u. angew. Math. 213 (1964); 89-102.

[2] Gociberg, I. A., and I. A. Fridmans: Faltungsgleichungen und Projektionsverfahren zu ihrer Lösung. Berlin 1974 .

[3] Grenander, U., and G. Szegö: Tóplitz Forms and Their Applications. Berkeley and Los Angeles 1958.

[4] Pomp, A.: Zur Konvergenź des Reduktionsverfahrens für Wiener-Hopfsche Gleichungen. Akad. der Wişs. der DDR, Inst. für Math.: Preprint P-Math-03/81 (Teil 1) und Preprint P-Math-05/81. (Teil 2).

[5̃] Prösssorf, S.: Einige Klàssen Singulärer Gleichungèn. Berlin 1974.

[6] Верьиций, И.Э., и Н. Я. КРупник: О применимости проекционного метода к дискретным уравнениям Винера-Хопфа с кусочно-непрерывным символом. Матем. Исслед. 45 (1977), 17-28.

[7] Levin, S.: On invertibility of finite sections of Toeplitz matrices. Applicable Analysis (im Druck).

Manuskripteingang: 17.02.1982 •

\section{VERFASSER:}

Dr. Andreas Pomp

Institut für Mathematik

der Akademie der Wissenschaften der DDR

DDR - 1080 Berlin, Mohrenstr. 39 